\title{
Validade dos Métodos Clínico e Ecográfico para 0 Diagnóstico de Adenomiose
}

\author{
Accuracy of Clinical and Ecographic Methods in the Diagnosis of Adenomyosis \\ Sérgio Fernandes Cabral Júnior, Hélio de Lima Fernandes Costa, Etevaldo Leite de Araújo, \\ Rosângela Pontes de Andrade, Simone Angélica Leite de Carvalho Silva Cabral
}

\begin{abstract}
RESUMO
Objetivos: determinar a sensibilidade, especificidade, valores preditivos positivo e negativo de um método clínico e da ultra-sonografia no diagnóstico da adenomiose.

Métodos: foi realizado estudo transversal do tipo validação de método diagnóstico incluindo 95 mulheres no menacme submetidas a histerectomia por diversas indicações. O diagnóstico clínico foi estabelecido em mulheres acima de 40 anos, dois ou mais filhos, com desvio menstrual para mais associado a dismenorréia. O diagnóstico ecográfico foi firmado pelo achado de, no mínimo, uma área mal delimitada de textura miometrial anormal hiper ou hipoecoíca, heterogênea ou cística. O critério histopatológico, considerado padrão-ouro, consistiu de achado de glândulas e estroma endometrial a $2,5 \mathrm{~cm}$ ou mais da junção endomiometrial.

Resultados: o método clínico apresentou sensibilidade de 68,2\%, especificidade de 78,1\%, valor preditivo positivo de 48,4\% e valor preditivo negativo de $89,1 \%$. Para o método ecográfico esses valores foram de, respectivamente, 45,5\%, 84,9\%, 47,6\% e 83,8\%. A razão de verosimilhança foi de 3,11 para o método clínico e 3,03 para o método ecográfico. Considerando apenas os casos positivos para ambos os métodos concomitantemente, a sensibilidade não ultrapassou 30\%, mas a especificidade aproximou-se de $100 \%$. Considerando-se os casos positivos em um dos dois métodos ou em ambos, a sensibilidade atingiu $86 \%$ e a especificidade a $60 \%$.

Conclusões: o método ecográfico não foi superior ao método clínico no diagnóstico de adenomiose.
\end{abstract}

PALAVRAS-CHAVE: Adenomiose. Endometriose. Histerectomia.

\section{Introdução}

A adenomiose é ginecopatia benigna, caracterizada pelo encontro de glândulas e estroma endometriais na intimidade do miométrio, associados ou não à hipertrofia e hiperplasia deste ${ }^{1,2}$. É doença que pode ser assintomática, mas que, na maioria das vezes, está ligada a sinais e sintomas como: menorragia, metrorragia, dismenorréia progressiva, útero de tamanho aumentado, dor

Disciplina de Tocoginecologia da Faculdade de Ciências Médicas da Universidade de Pernambuco - UPE Correspondência:

Hélio de Lima Ferreira Fernandes Costa

Rua Bruno Maia, 217, ap 1001 - Graças

52011-110 - Recife - PE pélvica crônica e dispareunia ${ }^{3,4}$. O quadro clínico, muitas vezes limitador das atividade habituais da mulher, é passivel de resolução definitiva somente por meio da histerectomia ${ }^{5}$.

O diagnóstico da adenomiose é sugerido, pela anamnese e exame físico, em mulheres na quarta e quinta décadas de vida que já pariram e/ou sofreram cirurgias sobre o miométrio e apresentam alterações clínicas como menorragia, dismenorréia e aumento do volume uterino ${ }^{6-8}$. O diagnóstico definitivo somente é firmado mediante a análise histopatológica do tecido miometrial obtido, usualmente, após a histerectomia ${ }^{1}$.

Somente na última década, os exames de ultra-sonografia endovaginal passaram a ser utilizados com o objetivo de tentar diagnosticar a ade- 
nomiose $\mathrm{e}^{-11}$. Esse exame complementar vem alcançando taxas de sensibilidade e especificidade de até $89 \%{ }^{12}$. Além disso, tem mostrado acurácia, em diagnosticar a adenomiose, semelhante à da ressonância magnética ${ }^{12,13}$, exame considerado como o mais fidedigno, todavia mais dispendioso e menos acessível ${ }^{2,14}$.

O emprego de métodos pré-cirúrgicos que demonstrem boa validade em diagnosticar a adenomiose é de fundamental importância à prática médica pois teremos a oportunidade de evitar tratamentos ineficazes, optando-se pela histerectomia quando a doença estiver presente ou tratamento conservador na sua ausência ${ }^{14,15}$.

O diagnóstico pré-operatório dessa doença ganha ainda maior importância quando levamos em consideração as mulheres que ainda guardam consigo o desejo de engravidar ou de preservar seu útero ou parte dele porque o consideram peça importante de sua feminilidade ${ }^{16}$.

O objetivo deste trabalho foi o de avaliar a validade dos métodos de diagnóstico clínico e ecográfico utilizados para o diagnóstico da adenomiose, testando-os isoladamente e comparando seus indicadores.

\section{Pacientes e Métodos}

Foi realizado estudo transversal de validação de método diagnóstico envolvendo 95 mulheres no menacme, internadas para se submeterem à histerectomia por causas diversas, no Serviço de Ginecologia do Centro Integrado de Saúde Amaury de Medeiros (CISAM) da Faculdade de Ciências Médicas (FCM) da Universidade de Pernambuco (UPE). As pacientes foram avaliadas no período de 15 de outubro de 1999 a 15 de dezembro de 2000. Foram excluídas do estudo mulheres em tratamento hormonal nos últimos dois meses anteriores ao internamento, virgens ou as que se encontravam no ciclo gravídico-puerperal.

Antes da cirurgia, as mulheres foram entrevistadas a respeito do seu passado obstétrico, sinais e sintomas ginecológicos vigentes e examinadas por meio de toque vaginal combinado, seguido de exame ecográfico transvaginal. Este foi realizado por dois ultra-sonografistas que não foram informados sobre os dados clínicos das pacientes. Utilizou-se equipamento ecográfico modelo Aloka Echocamera SSD-500, com transdutor vaginal de 5,0 $\mathrm{MHz}$.

O exame anatomopatológico da peça cirúrgica foi realizado no Departamento de Anatomopatologia do CISAM por única patologista, que executou um corte histopatológico em cada um dos seguin- tes locais do útero: fundo uterino, região ístmica, região ventral e dorsal. A avaliação do material foi feita com microscópio da marca Olympus - CDB, ao qual foi acoplado micrômetro graduado em décimo de milímetro, a fim de apurar a distância entre o foco miometrial suspeito de adenomiose e a junção endomiometrial. A patologista não foi informada do resultado do exame ecográfico realizado. Consideramos a adenomiose presente pelo método clínico em mulheres a partir de quarenta anos, que apresentassem desvio menstrual para mais (menorragia, hipermenorreia, hipermenorragia ou metrorragia), com dismenorréia e que tivessem no mínimo, dois filhos. A adenomiose foi considerada ausente na falta de pelo menos um dos critérios acima mencionados.

A adenomiose foi considerada presente, pelo método ecográfico, na presença de pelo menos uma área mal delimitada apresentando ecotextura miometrial anormal, de característica hiperecóica, hipoecóica, heterogênea ou cística (adenomiose difusa) ${ }^{11}$ ou área heterogênea circunscrita com margens indistintas, contendo lacuna anecóica de volume variável ${ }^{15}$

O diagnóstico histopatológico de adenomiose (padrão-ouro) ocorreu quando, na amostra do tecido miometrial, foram encontrados glândulas e estroma endometriais, distando, no mínimo, 2,5 mm da junção endomiometrial (Figura 1) ou quando se encontrou agregado nodular circunscrito de músculo liso e glândulas endometriais circundadas por hipertrofia miometrial com ou sem estroma (adenomioma) ${ }^{17}$.

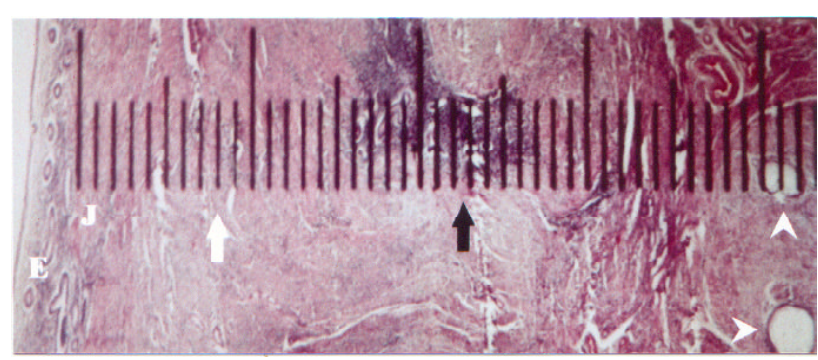

Figura 1 - Amostra de miométrio sob aumento de 40X, identificando-se endométrio (E) junção endomiometrial (J); invasão endomiometrial fisiológica (seta branca); foco de adenomiose atingindo $2,7 \mathrm{~mm}$ de profundidade (seta preta); e cistos miometriais a $4 \mathrm{~mm}$ de profundidade (cabeças de flechas).

A validade dos métodos clínico e ecográfico foi avaliada por meio dos seus respectivos valores de sensibilidade, especificidade, valores preditivos positivo e negativo. Além de avaliarmos a validade de cada método isoladamente, neste trabalho também confrontamos os indicadores de validade de cada método e utilizamos a razão de verossimilhança para testar a superioridade de qualquer dos 
métodos quanto à capacidade de diagnosticar corretamente a adenomiose.

O presente estudo avaliou também os indicadores de validade obtidos quando ambos os métodos foram combinados. Consideramos positividade para o método clínico e ecográfico apenas quando os dois testes são concomitantemente positivos. Por outro lado, consideramos positividade para o método clínico ou ecográfico quando um dos dois métodos ou ambos ao mesmo tempo resultem positivos.

O projeto de pesquisa foi aprovado pela $\mathrm{Co}^{-}$ missão de Ética em Pesquisa do CISAM (Centro Integrado de Saúde Amaury de Medeiros).

\section{Resultados}

Das 132 pacientes que aceitaram participar do estudo e satisfizeram aos critérios de inclusão e exclusão, 37 não foram incluídas na análise devido a: impossibilidade de avaliar adequadamente o miométrio pela presença de leiomiomas envolvendo mais de $50 \%$ do miométrio $(n=19)$; não-realização do exame ultra-sonográfico $(n=11)$; histerectomia não realizada $(n=4)$; exame histopatológico não realizado ou em desacordo com os critérios adotados pelo estudo $(n=3)$. Das 95 pacientes incluídas no estudo, 22 apresentaram diagnóstico histopatológico de adenomiose, correspondendo a uma prevalência de $23 \%$. A média de idade das pacientes com adenomiose $(45,6 \pm 4,4$ a) foi significativamente superior à das pacientes sem a doença $(40,9 \pm 5,5 \mathrm{a}, \mathrm{p}=0,0005)$. Não houve diferença estatisticamente significante em relação ao número de gestações $(3,8 \pm 2,3 \times 3,4 \pm 1,8)$ ou à paridade $(3,2 \pm 1,9 \times 2,8 \pm 1,6)$ entre os grupos.

A Tabela 1 mostra a avaliação da validade do critério clínico no diagnóstico da adenomiose. Este método foi capaz de diagnosticar quase $70 \%$ dos casos evidenciados ao exame histopatológico. A especificidade do método mostrou-se próxima a $80 \%$. O valor preditivo negativo (VPN) aproximouse dos 90\%, ao passo que o valor preditivo positivo (VPP) ficou abaixo de 50\%. Houve associação estatisticamente significante entre o método clínico e o diagnóstico histopatológico de adenomiose.

O método ecográfico teve sensibilidade inferior a 50\%, ao passo que sua especificidade atingiu quase $85 \%$ dos casos. Os VPP e VPN foram respectivamente de $47,6 \%$ e $83,8 \%$. Não houve associação estatisticamente significante entre o método ecográfico e o histopatológico de adenomiose (Tabela 2).

Foram comparados sensibilidade, especificidade, VPP e VPN de ambos critérios adotados visando diagnosticar a adenomiose. Dada a superposição dos intervalos de confiança, não foram encontradas diferenças estatisticamente significantes entre os indicadores de validade dos métodos. As razões de verossimilhança de ambos os métodos foram semelhantes entre si, respectivamente 3,11 e 3,03 para o clínico e o ecográfico. Estes dados são encontrados na Tabela 3.

Tabela 1 - Distribuição das pacientes submetidas a histerectomia abdominal de acordo com o diagnóstico de adenomiose pelo método clínico e pelo método histopatológico.

\begin{tabular}{lcccc}
\hline \multicolumn{5}{c}{ Diagnóstico Histopatológico } \\
Método & Presente & Ausente & Total & $\mathbf{p}^{*}$ \\
clínico & $\mathbf{n}(\%)$ & $\mathbf{n ~ ( \% )}$ & $\mathbf{n}(\%)$ & \\
\hline Presente & $15(68,2)$ & $16(21,9)$ & $31(32,6)$ & 0,0001 \\
Ausente & $7(31,8)$ & $57(78,1)$ & $64(67,4)$ & \\
Total & 22 & 73 & 95 & \\
Sensibilidade: $68,2 \%$ & Especificidade: $78,1 \%$ & VPP: $48,4 \%$ & VPN: $89,1 \%$ &
\end{tabular}

*? ${ }^{2}$ com correção Yates

Tabela 2 - Distribuição das pacientes submetidas a histerectomia abdominal de acordo com o diagnóstico de adenomiose pelo método ecográfico e pelo método histopatológico.

\begin{tabular}{|c|c|c|c|c|}
\hline \multirow[b]{2}{*}{$\begin{array}{l}\text { Método } \\
\text { ecográfico }\end{array}$} & \multicolumn{3}{|c|}{ Diagnóstico Histopatológico } & \multirow[b]{2}{*}{$p^{*}$} \\
\hline & $\begin{array}{c}\text { Presente } \\
\text { n (\%) }\end{array}$ & $\begin{array}{c}\text { Ausente } \\
\text { n (\%) }\end{array}$ & $\begin{array}{l}\text { Total } \\
\text { n (\%) }\end{array}$ & \\
\hline Presente & $10(45,5)$ & $11(15,1)$ & $21(22,1)$ & $0,206^{*}$ \\
\hline Ausente & $12(54,5)$ & $62(84,9)$ & $74(77,9)$ & \\
\hline Total & 22 & 73 & 95 & \\
\hline
\end{tabular}

Sensibilidade: 45,5\% Especificidade: 84,9\% VPP: 47,6\% VPN: 83,8\%

*? ${ }^{2}$ com correção Yates

Tabela 3 - Comparação dos indicadores de validade do método clínico com os indicadores do método ecográfico para o diagnóstico de adenomiose.

\begin{tabular}{lcccc}
\hline & $\begin{array}{c}\text { Método } \\
\text { clínico }\end{array}$ & IC & ecográfico & IC \\
\hline Sensibilidade & $68,2 \%$ & $45,1-85,3$ & $45,5 \%$ & $25,1-67,3$ \\
Especificidade & $78,1 \%$ & $66,6-86,6$ & $84,9 \%$ & $74,2-91,9$ \\
VPP & $48,4 \%$ & $30,6-66,6$ & $47,6 \%$ & $26,4-69,7$ \\
VPN & $89,1 \%$ & $78,2-95,1$ & $83,8 \%$ & $73,0-91,0$ \\
Razão de & & & & \\
Verossimilhança & 3,11 & & 3,03 & \\
Valor do p & 0,0001 & & 0,206 & \\
\hline
\end{tabular}

Avaliando-se os indicadores de validade obtidos quando os métodos foram combinados e considerando a adenomiose presente quando identificada por ambos os testes ao mesmo tempo, a sensibilidade não ultrapassou $30 \%$, ao passo 
que a especificidade aproximou-se de 100\%. Quando os dois métodos são utilizados combinadamente, considerando-se positivos todos os casos diagnosticados por um dos dois métodos ou por ambos concomitantemente, conseguiu-se diagnosticar corretamente quase $86 \%$ dos casos de adenomiose e um pouco mais de $60 \%$ dos casos sem esta doença (Tabela 4).

Tabela 4 - Distribuição das pacientes submetidas a histerectomia abdominal de acordo com o diagnóstico de adenomiose pela combinação do método clínico e/ou ecográfico e com o diagnóstico histopatológico de adenomiose.

\begin{tabular}{|c|c|c|c|}
\hline \multirow[t]{2}{*}{$\begin{array}{l}\text { Método combinado } \\
\text { clínico e ecográfico }\end{array}$} & \multicolumn{3}{|c|}{$\begin{array}{c}\text { Diagnóstico } \\
\text { histopatológico de adenomiose }\end{array}$} \\
\hline & $\begin{array}{l}\text { Presente } \\
\text { n (\%) }\end{array}$ & $\begin{array}{l}\text { Ausente } \\
\text { n (\%) }\end{array}$ & $\begin{array}{l}\text { Total } \\
\text { n }(\%)\end{array}$ \\
\hline \multicolumn{4}{|l|}{ Método combinado } \\
\hline \multicolumn{4}{|l|}{ Cínico e ecográfico } \\
\hline Presente & $6(27,3)$ & $1(1,4)$ & $7(7,4)$ \\
\hline Ausente & $16(72,7)$ & $72(98,6)$ & $88(92,6)$ \\
\hline \multicolumn{4}{|l|}{ Método combinado } \\
\hline \multicolumn{4}{|l|}{ Clínico ou ecográfico } \\
\hline Presente & $19(86,4)$ & $26(35,6)$ & $45(47,4)$ \\
\hline Ausente & $3(13,6)$ & $47(64,4)$ & $50(52,6)$ \\
\hline Total & 22 & 73 & 95 \\
\hline $\begin{array}{l}\text { Método combinado cínico e } \\
\text { Sensibilidade: } 27,3 \% \\
p=0,0005 \text { (Fisher) } \\
\text { Método combinado cínico o }\end{array}$ & $\begin{array}{l}\text { e ecográfico } \\
\text { Especificidade: } 98,6 \% \\
\text { ou ecográfico }\end{array}$ & VPP: $85,7 \%$ & VPN: $81,8 \%$ \\
\hline $\begin{array}{l}\text { Sensibilidade: } 86,4 \% \\
p=0.0008\left(?^{2}\right)\end{array}$ & Especificidade: 64,4\% & VPP: $42,2 \%$ & VPN: $94,0 \%$ \\
\hline
\end{tabular}

\section{Discussão}

A definição de um método simples, seguro e acurado para o diagnóstico da adenomiose é questão relevante na Ginecologia. Embora as manifestações clínicas da adenomiose sejam bem conhecidas não há critério de diagnóstico clínico objetivamente definido e consensualmente aceito. No caso da ultra-sonografia, por sua vez, trata-se de exame complementar simples, não invasivo e pouco oneroso, que vem se firmando como método promissor para este fim ${ }^{11,13,18}$.

$\mathrm{Na}$ presente amostra, o método clínico foi capaz de diagnosticar quase $70 \%$ dos casos de adenomiose comprovados ao exame histopatológico. Não encontramos na literatura consultada método diagnóstico clínico similar, contra o qual pudéssemos confrontar nossos resultados. Todavia, se levarmos em consideração a sensibilidade do diagnóstico clínico presuntivo para a adenomiose apresentado por diversos autores entre 0\% e $28 \%$, ${ }^{6,19}$ o nosso estudo mostrou taxa significativamente mais elevada $(68,2 \%)$.

Acreditamos que obtivemos melhor sensibilidade pelo fato de termos elaborado e utilizado método clínico composto de variáveis que, apesar de sugestivas de adenomiose $\mathrm{e}^{3,4}$, até o momento não foram empregadas como critério clínico definido com o objetivo de diagnosticar essa enfermidade. Ademais, a seleção de mulheres exclusivamente no menacme pode ter facilitado o diagnóstico clínico, visto que, na pós-menopausa o foco adenomiótico torna-se atrófico e assintomático ${ }^{20}$.

Neste trabalho, não pudemos avaliar mulheres submetidas à histerectomia apenas por prolapso uterino, pois todas estas eram menopausadas. Esse fato também poderia ter contribuído para alavancar a nossa taxa de acerto. Em estudo multicêntrico Lee et al. $(1984)^{21}$ notaram que apenas 9\% das mulheres histerectomizadas exclusivamente por relaxamento pélvico possuíam adenomiose, ao passo que Blum (1981) ${ }^{22}$ mostrou $12 \%$ de adenomiose em grupo semelhante de pacientes.

Não foi identificada em nosso estudo qualquer área sugestiva de adenomioma ao exame ecográfico endovaginal, e, portanto foram avaliados apenas casos de adenomiose difusa.

O método ultra-sonográfico apresentou sensibilidade de $45,5 \%$ e especificidade de $84,4 \%$ na amostra estudada. A primeira ficou bem abaixo das taxas encontradas por diversos autores, que citam cifras entre 80 e $89 \%^{9,12}$, ao passo que a segunda manteve-se dentro da faixa entre $74 \%$ e $92,2 \%$, relatada na maioria dos estudos ${ }^{9,18}$.

O VPP $(47,6 \%)$ ficou bem abaixo dos valores relatados por vários autores que variam de $71 \%$ a $86 \%^{10,12}$, ao passo que o VPN $(83,1 \%)$ ficou dentro da variação apontada por outros pesquisadores $(81 \% \text { a } 98,6 \%)^{9,15}$. Este último resultado chama a atenção, pois quando um método diagnóstico alcança bom VPN é capaz de evitar cirurgias desnecessárias, apontando corretamente casos sem adenomiose ${ }^{11}$.

Um dos fatores limitantes da validação do método ecográfico residiu na baixa resolução do aparelho de ultra-sonografia que utilizamos. Esse instrumento diagnóstico pode não ter sido capaz de diagnosticar focos de adenomiose que, de acordo com Reinhold et al. (1995) ${ }^{11}$, podem ser sutis e não perceptiveis por esse exame complementar.

Mais de 70\% das mulheres selecionadas possuíam leiomiomas. Essa enfermidade pode ter contribuído para dificultar o diagnóstico ecográfico da adenomiose, conforme defendem Hirai et al. ${ }^{23}$. Além disso, a presença de calcificações vasculares, hiperplasia muscular idiopática, alterações 
linfáticas e "artefatos" podem contribuir para o aumento do número de falso-positivos com esse tipo de exame complementar ${ }^{7,11}$.

O exame ultra-sonográfico depende do operador, podendo haver diferenças de diagnósticos conforme tempo de experiência do ultra-sonografista $^{11,24}$. Isso pode ter influenciado negativamente nos resultados da ecografia, na medida que utilizamos em nosso estudo análises de dois ultrasonografistas. Por outro lado, a ultra-sonografia foi realizada meticulosamente em tempo real e não a partir de fotografias. Este último tipo de avaliação pode diminuir a capacidade de identificação das imagens sutis sugestivas de adenomiose ${ }^{2}$.

Inicialmente, ao avaliarmos a acurácia de ambos os métodos, tendemos a concluir que o método clínico foi melhor que o ecográfico. Todavia, ao compararmos a razão de verossimilhança dos métodos, percebemos que eles possuem resultados muito próximos, respectivamente 3,11 para o método clínico e 3,03 para o ecográfico. Esses resultados indicam que nenhum método foi superior ao outro de modo estatisticamente significante.

De acordo com o guia elementar de interpretação elaborado por Jaeschke et al. ${ }^{25}$, os valores de verossimilhança que se situam entre 2 e 5 geram pequenas variações de probabilidade pré e pós-teste. Assim, a probabilidade de uma pessoa ter realmente adenomiose, uma vez identificada com os métodos estudados, não foi muito diferente da estimativa antes da realização dos testes.

Ao utilizarmos a combinação dos dois métodos, considerando diagnóstico de adenomiose a positividade clínica e/ou ecográfica, a elevada sensibilidade $(86 \%)$ sugere que poucos casos de adenomiose não serão detectados quando os dois métodos são empregados. Desta forma, um valor preditivo negativo de 94\% reforça a opção de tentar tratamento conservador para a pacientes negativas para ambos os métodos.

Por outro lado, a combinação dos métodos considerando diagnóstico de adenomiose a positividade clínica e ecográfica concomitantemente revelou ótima especificidade, o que, ao nosso ver reflete apenas a fraca capacidade de identificação da afecção por essa associação diagnóstica.

Concluímos que o método de diagnóstico ecográfico não foi superior ao clínico quanto à capacidade de identificar a adenomiose, e que, boa sensibilidade pode ser conseguida quando a doença é diagnosticada por um ou outro teste empregados em conjunto. Destarte, o diagnóstico histopatológico não pode ser substituído pelos métodos clínico ou ecográfico, na forma como utilizados no presente estudo.

Recomendamos que novas pesquisas, visando avaliar a acurácia clínica e ecográfica dessa enfermidade devam ser realizadas. Utilizando maior tamanho amostral e instrumento ecográfico de melhor resolução, melhores resultados poderão ser atingidos, proporcionando à mulher tratamento mais esclarecido e adequado aos seus interesses.

\section{ABSTRACT}

Purpose: to evaluate the sensitivity, specificity, positive and negative predictive values of a clinical and an ecographic method for adenomyosis diagnosis.

Methods: a transversal study of validation of the diagnostic method was done, including 95 women in menacme submitted to hysterectomy for various causes. Adenomyosis was diagnosed through a clinical method in women aged 40 years or older, with 2 or more deliveries, increased menstrual bleeding associated with dysmenorrhea. The ecographic diagnosis was established if at least one myometrial ill defined area of abnormal ecotexture was found, which could be hypoechoic, hyperchoic, heterogeneous or cystic. Gold standard was histopathology, defined as the finding of endometrial glands or stroma more than $2.5 \mathrm{~cm}$ above the endomiometrial junction.

Results: the clinical method had $68.2 \%$ sensitivity, $78.1 \%$ specificity, $48.4 \%$ positive predictive value and $89.1 \%$ negative predictive value. For the echographic method this figures were, respectively, 45.5\%, 84.9\%, $47.6 \%$ and $83.8 \%$. Likelihood ratio was 3.11 for the clinical and 3.03 for the echographic method. Considering only those simultaneously positive cases by both methods, sensitivity was below $30 \%$ and specificity was near 100\%. Considering all positive cases by one or the other method or concomitanty by both, the sensitivity reached $86 \%$ and specificity was $60 \%$.

Conclusion: the echographic method was not better than the clinical for the diagnosis of adenomyosis.

KEY WORDS: Adenomyosis. Endometriosis. Hysterectomy.

\section{Referências}

1. Hendrickson MR, Kempson RL. Non-neoplastic conditions of the myometrium and uterine serosa. In: Fox H, Wells M, editors. Haines and Taylor Obstetrical and Gynaecological Pathology. $4^{\text {th }}$ ed. New York: Churchill Livingstone;1995. p.511-7.

2. Reinhold C, Tafazoli F, Mehio A, et al. Uterine adenomyosis: endovaginal US and MR imaging features with histopathologic correlation. Radiographics 1999; 19 Spec No:S147-60. 
3. Ferenczy A. Pathophysiology of adenomyosis. Hum Reprod Update 1998; 4:312 - 22.

4. Levgur M, Abadi MA, Tucker A. Adenomyosis: symptoms, histology, and pregnancy terminations. Obstet Gynecol 2000; 95:688-91.

5. Kistner RW. Tratado de Ginecología. $2^{\mathrm{a}}$ ed. Barcelona: Toray; 1983.p.222-6.

6. Vavilís D, Agorastos T, Tzafetas J, et al. Adenomyosis at hysterectomy: prevalence and relationship to operative findings and reproductive and menstrual factors. Clin Exp Obstet Gynecol 1997; 24:36-8.

7. Azziz R. Adenomyosis: current perspectives. Obstet Gynecol Clin North Am 1989; 16:221-35.

8. Siegler AM, Camilien L. Clinical perspectives. Adenomyosis. J Reprod Med 1995; 39:841-53.

9. Fedele L, Bianchi S, Dorta M, Arcaini L, Zanotti, F, Carinelli S. Transvaginal ultrasonography in the diagnosis of diffuse adenomyosis. Fertil Steril 1992; 58:94-7.

10.Brosens JJ, de Souza NM, Barker FG, Paraschos TI, Winston RM. Endovaginal ultrasonography in the diagnosis of adenomyosis uteri: identifying the predictive characteristics. Br J Obstet Gynaecol 1995; 102:471-4.

11.Reinhold C, Atri M, Mehio A, Zakarian R, Aldis AE, Bret PM. Diffuse uterine adenomyosis: morphologic criteria and diagnostic accuracy of endovaginal sonography. Radiology 1995; 197:609-14.

12.Reinhold C, McCarthy S, Bret PM, et al. Diffuse adenomyosis: comparison of endovaginal US and MR imaging with histopathologic correlation. Radiology 1996; 199:151-8.

13.Bazot M, Cortez A, Darai E, et al. Ultrasonography compared with magnetic resonance imaging for the diagnosis of adenomyosis: correlation with histopathology. Hum Reprod 2001; 16:2427-33.

14.Botsis D, Kassanos D, Antoniou G, Pyrgiotis E, Karakitsos P, Kalogirou D. Adenomyoma and leiomyoma: differential diagnosis with transvaginal sonography. J Clin Ultrasound 1998; 26:21-5.
15.Fedele L, Bianchi S, Dorta M, Zanotti F, Brioschi D, Carinelli S. Transvaginal ultrasonography in the differential diagnosis of adenomyoma versus leiomyoma. Am J Obstet Gynecol 1992; 167:603-6.

16. Wood C. Surgical and medical treatment of adenomyosis. Hum Reprod Update 1998; 4:323-36.

17.Zaloudeck C, Norris HJ. Mesenchymal tumors of the uterus. In: Kurman RJ, editor. Blaustein's Pathology of the Female Genital Tract. $1^{\text {st }}$ ed. New York: Springer-Verlag; 1994.p.518.

18.Atzori E, Tronci C, Sionis L. Transvaginal ultrasound in the diagnosis of diffuse adenomyosis. Gynecol Obstet Invest 1996; 42:39-41.

19.Thomas JS Jr, Clark, JF. Adenomyosis: a retrospective view. J Natl Med Assoc 1989; 81:96972 .

20.Hesla JS, Rock JA. Endometriose. In: Rock, JA, Thompson JD, editores. Te Linde Ginecologia Operatória. $8^{\mathrm{a}}$ ed. Rio de Janeiro: Guanabara Koogan; 1999. p.484-7.

21.Lee NC, Dicker RC, Rubin GL, Ory HW. Confirmation of the preoperative diagnosis for hysterectomy. Am J Obstet Gynecol 1984; 150:2837.

22.Blum M. Adenomyosis: study in a Jewish female population. Int Surg 1981; 66:341-3.

23.Hirai M, Shibata K, Sagai H, Sekiya S, Goldberg BB. Transvaginal pulsed and color Doppler sonography for the evaluation of adenomyosis. J Ultrasound Med 1995; 14:529-32.

24.Atri M, Reinhold C, Mehio AR, Chapman WB, Bret PM. Adenomyosis: US features with histologic correlation in an in-vitro study. Radiology 2000; 215:783-90.

25.Jaeschke R, Guyatt G, Sackett DL. Users' guides to the medical literature. III. How to use an article about a diagnostic test. A. Are the results of the study valid? Evidence-Based Medicine Working Group. JAMA 1994; 271:389-91. 